\title{
Initiatives de développement économique communautaire dans quatre métropoles nord-américaines ${ }^{1}$
}

\author{
Jean-Marc Fontan - Université du Québec à Montréal \\ Pierre Hamel - Université de Montréal \\ Richard Morin - Université du Québec à Montréal \\ Eric Shragge - Université Concordia ${ }^{2}$
}

\section{Introduction}

La réalité du développement économique communautaire en milieu métropolitain nord-américain est mal connue. Cet article apporte des éléments d'éclairage en se basant sur les conclusions d'une recherche que nous venons de terminer. Nous avons étudié les modalités de cohabitation entre des stratégies de développement économique reposant sur une logique métropolitaine d'intervention et celles fondées sur une approche locale et communautaire.

La recherche portait sur quatre métropoles de l'Est du continent Nord-Américain: Boston, Montréal, Toronto et Pittsburgh. Ces villes ont été retenues du fait qu'elles appartiennent à une zone territoriale de proximité relative et qu'elles sont de poids démographique semblable. Les quatre villes ont une tradition de développement communautaire et elles ont fait l'objet de stratégies de développement économique.

\section{Deux grandes questions ont traversé} la recherche que nous avons menée 3 . En premier lieu, nous voulions caractériser les quatre contextes métropolitains d'intervention pour comprendre les convergences et les divergences auxquels les acteurs en développement sont confrontés. En deuxième lieu, nous voulions voir si les interventions par le bas et par le haut s'actualisaient en vase clos ou si elles entretenaient des liens forts, sinon des liens faibles, les unes avec les autres. Nous présentons ici les résultats découlant de la deuxième interrogation.
Deux grandes questions ont traversé la recherche que nous avons menée ${ }^{3}$. En premier lieu, nous voulions caractériser les quatre contextes métropolitains d'intervention pour comprendre les convergences et les divergences auxquels les acteurs en développement sont confrontés. En deuxième lieu, nous voulions voir si les interventions par le bas et par le haut s'actualisaient en vase clos ou si elles entretenaient des liens forts, sinon des liens faibles, les unes avec les autres. Nous présentons ici les résultats découlant de la deuxième interrogation.

$\mathrm{Au}$ terme de la recherche, nous sommes en mesure d'observer une situation paradoxale. Malgré la présence, dans chaque métropole étudiée, d'une pratique de développement économique communautaire, malgré une présence généralisée dans les discours politiques états-unien, canadien et québécois de mentions indiquant l'importance de faire reposer le développement sur l'ensemble des acteurs métropolitains, il appert que l'actualisation de ce discours est faiblement ou nullement prise en considération par les leaders des métropoles étudiées. Dans chaque métropole, nous avons observé que le rôle de l'État fédéral et de l'État «régional» est central dans le soutien accordé aux initiatives de développement économique communautaire. Nous avons aussi observé que la prise en considération du développement économique communautaire par les acteurs métropolitains est faible quand elle n'est pas inexistante.

Il se dégage alors de notre étude le sentiment que les grands acteurs métropolitains sont plus préoccupés de la croissance de leur économie que de la généralisation d'une partie de ce développement à l'ensemble de la population. Ils abandonneraient à l'État régional et à l'État fédéral la responsabilité de soutenir les initiatives 
novatrices à vocation providentielle. Ils ne se soucieraient dès lors pas des tensions et des problèmes que suscite une politique non intégrée de croissance socioéconomique de leur territoire.

\section{Il se dégage alors de notre étude le sentiment que les grands acteurs métropolitains sont plus préoccupés de la croissance de leur économie que de la généralisation d'une partie de ce développement à l'ensemble de la population.}

\section{Initiatives locales de développement et de gouvernance}

Il ne nous est pas possible de présenter un portrait d'ensemble de l'évolution et de l'état des initiatives locales pour les quatre métropoles étudiées. Nous avons choisi de limiter notre analyse à l'étude des initiatives vouées au développement économique et à la gouvernance locale préconisant une approche communautaire.

Le développement économique communautaire émerge aux États-Unis et au Canada à partir des années 1960. Cette stratégie d'intervention est implantée dans des quartiers urbains soumis à des contraintes importantes en termes de sous-développement de leur capacité productive. D'une part, les activités économiques y sont en déclin ou peu présentes. D'autre part, leur population active connaît des problèmes sérieux d'intégration sur le marché du travail. Il s'ensuit un processus d'appauvrissement propice à l'émergence d'une culture de la pauvreté au sein de la population habitant ces quartiers.

Dans les quatre villes étudiées, le développement économique communautaire, défini comme une stratégie d'intervention proposant une prise en charge du développement de zones marginalisées par des représentants de ces communautés, fait son apparition à la fin des années 1960 ou au début des années 1970. Par contre, les organisations dotées de ressources importantes et d'une mission de revitalisation socio-économique des territoires, connues sous le nom de «Community Development Corporations» (CDC), se mettent en place dans les villes des États-Unis au milieu des années 1960, plus particulièrement sur les côtes est (New York) et ouest
(Los Angeles) du pays. Elles apparaissent plus tardivement au Canada, c'est-à-dire vers la fin des années 1970 en Nouvelle-Écosse et au début des années 1980 au Québec et en Ontario (au départ, à Montréal et à Toronto). $\mathrm{Au}$ Québec, ces organisations sont connues sous le vocable de «Corporations de développement économique communautaires» (CDÉC).

Une première particularité des CDC et des CDÉC est de proposer aux divers représentants des différentes instances gouvernementales une nouvelle philosophie en termes d'intervention publique relativement à la revitalisation de quartiers et de communautés en difficulté. À l'approche supplétive reposant sur l'allocation providentielle de mesures de sécurité du revenu, ces organismes mettent de l'avant une approche complémentaire et pro active de soutien aux initiatives qui redonneraient aux personnes et aux collectivités locales une prise sur leur capacité d'améliorer leur situation, les aidant à sortir du cercle de la pauvreté. Les dispositifs utilisés pour amorcer «une prise en charge» du développement par les acteurs locaux renvoient à une variété de modèles d'intervention: principalement la création d'emplois dans le cas de Montréal, le logement dans le cas des États-Unis, ou les deux dans le cas de Toronto.

Une deuxième particularité de ces organisations est de proposer une synergie entre les différents intervenants chargés de soutenir les initiatives locales de développement. C'est ce qui nous conduit à introduire la question de la gouvernance locale. En effet, le travail de concertation et de partenariat à l'intérieur duquel s'engagent ces organisations consiste à intervenir de concert avec de nombreux acteurs économiques et institutionnels afin d'influencer les lieux décisionnels clés. Les quatre villes étudiées présentent des expériences innovantes en matière de concertation et de partenariat entre les instances municipales et des représentants des gouvernements régionaux (États et provinces) incluant parfois des commerçants ou des entrepreneurs locaux.

À Boston, par exemple, les représentants de la CDC d'un des quartiers les plus marginalisés de la ville, le quartier Roxbury, ont obtenu de la part de la Ville le droit d'exproprier des propriétaires délinquants afin de reprendre possession de tout terrain laissé délibérément à l'abandon, mais dont le propriétaire ne voulait pas se départir en raison d'intérêts spéculatifs. À Montréal, la Société de développement Angus, mise en place par une 
CDÉC, a réussi un partenariat inédit avec des représentants du secteur privé et des instances des trois paliers de gouvernement pour revitaliser une partie de la friche industrielle Angus. À Toronto, le Learning Enrichment Foundation (LEF) gère un budget de seize millions de dollars pour assurer une intervention très diversifiée allant de formations vouées au développement de l'employabilité à des services communautaires de différents types, en passant par l'administration d'un incubateur d'entreprises et un service de soutien aux nouveaux entrepreneurs. L'intervention de la LEF s'appuie sur la collaboration de plusieurs acteurs: organismes communautaires oeuvrant dans divers domaines, élus et fonctionnaires des divers paliers de gouvernement, corporations et fondations privées. Enfin, à Pittsburgh, une CDC, la Manchester Citizens Corporation, a permis l'investissement de plus de quarante millions de dollars U.S. dans des projets de revitalisation immobilière, depuis sa création en 1965. À cet égard, la corporation est engagée dans un partenariat avec une banque locale qui lui permettra d'élargir son intervention au domaine de la formation à l'emploi et de support au développement d'entreprises.

Une troisième particularité de ces expériences, moins généralisée toutefois que les deux précédentes, consiste en la capacité des CDC et des CDÉC de se regrouper au sein de coalitions pour négocier ou transiger avec les représentants du secteur public. C'est le cas à Montréal, avec l'Inter-CDÉC (qui regroupe une douzaine de CDÉC de l'île de Montréal), à Pittsburgh, avec le Pittsburgh Community Reinvestment Group (qui comprend 29 CDC) et le Pittsburgh National Alliance (qui rassemble 80 organisations communautaires) et à Boston, avec le Community Business Network et le Neighborhood Means Business. À Toronto, de tels regroupements n'ont pas vu le jour. Dans cette ville, les leaders du mouvement de développement économique communautaire ont tenté à plusieurs reprises de former une coalition au sein d'une organisation parapluie, mais sans succès.

Enfin, le bourgeonnement des expériences de développement local à caractère progressiste témoigne de l'existence de modalités distinctes de financement de ces organisations au Canada et aux États-Unis. La place des fondations privées est très grande dans le financement des CDC états-uniennes, alors qu'elle est minime dans les expériences canadiennes en général et embryonnaire dans les expériences québécoises et montréalaises. Dès lors, les relations que ces initiatives entretiennent avec
l'État prennent une importance accrue à Toronto et surtout à Montréal. Toutefois, quand l'État intervient à Boston ou à Pittsburgh, force est de constater que cette action est soutenue par des financements partagés provenant principalement de programmes mis sur pied par les paliers publics régionaux et nationaux.

\section{Gouvernance métropolitaine et arrimage aux initiatives locales}

Les métropoles étudiées et les initiatives locales dont il vient d'être question s'insèrent dans des cadres institutionnels très différents. Les États-Unis et le Canada ont une structure de régulation politique partagée entre les institutions de l'État fédéral, de l'État régional ou de la province, de la zone métropolitaine et de la municipalité. Toutefois, les rôles et les fonctions réparties entre ces paliers de régulation sont différents d'un pays à l'autre. De plus, comme nous l'avons déjà mentionné, les CDC aux États-Unis bénéficient d'un financement substantiel en provenance de fondations privées, ce qui influence aussi le cadre de contraintes auxquelles ces organisations doivent répondre pour obtenir leur financement. Peu d'études se sont penchées sur l'impact de ce partenariat de type privé sur le développement local orienté vers une solidarité à caractère social et communautaire. Aux États-Unis, cette situation existe depuis très longtemps. Au Canada, elle prend un peu plus d'importance depuis une vingtaine d'années (d'ailleurs plus à Toronto qu'à Montréal). De ce foisonnement institutionnel, il importe de mieux cerner le rôle des institutions sur lesquelles reposent la gouvernance et le développement métropolitains de même que celles qui sont directement en lien avec les organismes de développement économique communautaires. Le degré d'arrimage entre les institutions de gouvernance métropolitaine et les organismes de DEC nous apparaît aussi un nouvel enjeu des plus significatif dans le présent contexte de mondialisation.

Pour la ville de Boston, il existe une institution qui intervient principalement auprès des CDC. Il s'agit du Department of Neighbourhood Development. Ce département est divisé en différents services dont certains sont en interaction directe avec les CDC, tels l'Office of Business Development et le Boston Main Streets. Par contre, il n'existe aucune structure métropolitaine d'intervention, à l'instar de la Boston Redevelopment Authority, uniquement chargée des grands travaux comme l'immense chantier de reconstruction de l'auto- 
route qui traverse la ville (le Big Dig comme on dit à Boston) qui aurait pour fonction de mettre en place des programmes d'aide ou d'intervenir auprès des organismes communautaires. Une séparation nette est observable entre les interventions liées au soutien des initiatives issues de «l'économie des ligues majeures» et celles associées à «l'économie des ligues mineures» fondée sur la proximité. Il convient ici de signaler qu'à Boston, la gouvernance métropolitaine, c'est-à-dire la collaboration entre des acteurs issus du secteur public et du secteur privé, à des fins de développement métropolitain, ne s'incarne pas dans une organisation de type partenariale. Certes, nous retrouverons à Boston un organisme tel que le Boston's Metropolitan Area Planning Council (MAPAC), mais il s'agit davantage d'un forum de discussion que d'une véritable institution de gouvernance. De plus, même si le gouvernement de l'État offre des services aux entreprises par le biais du Massachusetts Office of Business Development (MOBD) qui possède un bureau dédié au Boston métropolitain, celui-ci n'entretient aucun contact direct avec les CDC.

Les CDC de Boston peuvent toutefois compter sur un apport financier important en provenance de l'État fédéral. Ce dernier a mis en place différents outils dont le Small Business Administration et le Department of Housing and Urban Development (HUD). C'est ce département qui est responsable du financement des CDC. C'est ce qui explique aussi pourquoi la majorité des CDC se spécialisent dans le domaine de l'immobilier résidentiel ou commercial à vocation sociale. Dans le cas particulier de Boston, le HUD a consenti à la ville l'application du programme Enhanced Enterprise Community (EEC) pour revitaliser un groupe de quartiers défavorisés, dont ceux de Roxbury et Dorchester Nord. Le programme se veut global puisqu'il vise le développement de l'emploi et la formation à l'emploi ainsi que le développement des programmes d'éducation des adultes, des services d'aide à la petite enfance et de support aux enfants qui ont décroché du système scolaire, d'aide au démarrage d'entreprises et du support pour la revitalisation immobilière. Le conseil d'administration de la corporation chargée de la mise en œuvre du programme est «communautaire» au sens où les représentants proviennent des différents milieux des communautés concernées et des leaders politiques de la ville de Boston.

Dans le cas de la région de Pittsburgh, dès les années 1930 et 1940, les leaders du secteur privé ont pris l'ini- tiative de se regrouper pour mener des études sur la région et élaborer des stratégies de développement. D'abord, la Pennsylvania Economy League (PEL) comprenant six divisions, dont une consacrée à la grande région de Pittsburgh, est mise sur pied en 1936. Ensuite, la Allegheny Conference on Community Development (ACCD) est créée en 1943. La ACCD a joué un rôle important en matière de développement en mobilisant le secteur privé et le secteur public autour d'une série de plans de revitalisation urbaine. Le premier plan Renaissance a vu le jour en 1945 et s'est appliqué jusqu'en 1970. Le deuxième plan a été amorcé en 1978 et a prévalu jusqu'en 1982 alors que le troisième est en cours depuis 1982.

Le plan Renaissance I s'inscrit dans la ligne des projets de rénovation urbaine de l'après Deuxième Guerre mondiale. Il s'agit de grands projets pensés par le haut et gérés par une corporation nouvellement créée, la Urban Redevelopment Authority of Pittsburgh (URA). Celle-ci relève du maire de la ville et d'un conseil de direction qui lui est propre. L'URA vise avant tout le développement des infrastructures métropolitaines. Peu de place est accordée à la concertation élargie, si ce n'est celle entre les élites économiques et politiques. Le plan Renaissance II, quant à lui, fait appel à une autre logique de développement. L'approche est plus sociale-démocrate. La concertation est élargie et les modalités de gouvernance de Renaissance I sont revues afin d'intégrer les voies dissidentes qui s'opposaient à la façon dont fut géré le plan Renaissance I par l'URA. À titre indicatif du rapprochement effectué avec les organisations locales de développement communautaire, un partenariat est conclu entre l'URA et la CDC Manchester Citizens Corporation pour des projets de revitalisation résidentielle.

Cependant, le plan Renaissance III a effectué un retour en arrière en proposant un resserrement politique qui permet de revenir à la logique de développement par le haut. L'objectif est de renouer avec les grands projets de développement, dont celui d'attirer à Pittsburgh des entreprises de haute technologie. Le plan Renaissance III donne lieu à une concertation élargie entre des institutions de la zone métropolitaine de Pittsburgh et celles des autres zones urbaines limitrophes. Différents projets émanent de cette concertation élargie aux deux grandes universités de la région, tel le plan d'action Strategy 21 qui vise le renforcement ou le développement de grandes infrastructures (dont la modernisation de l'aéroport, l'implantation d'une stratégie de développement touristique et la reconversion des friches indus- 
trielles). Cette perspective, définie en termes de nouvelles modalités de développement pour la région s'est traduite dernièrement par la création de la Pittsburgh Regional Alliance qui regroupe les cinq grands organismes publics de développement de la grande région de Pittsburgh (Champagne, 1998). Il s'agit là en fait d'une démarche de planification qui remonte au milieu des années 1985 à partir d'une vision d'intégration de la région métropolitaine de Pittsburgh aux grands réseaux d'affaires de la mondialité. Le support aux organisations locales et communautaires n'est pas complètement abandonné, mais il ne fait aucunement partie des grands axes d'intervention stratégique. Là encore, la séparation entre de grandes actions structurantes de développement et de petites actions de revitalisation misant sur l'économie de proximité est apparente. Les CDC sont appuyées pour les actions qu'elles mènent dans leur quartier respectif mais elles ne jouent pas de rôle significatif en ce qui concerne les stratégies de développement métropolitain.

L'hypothèse de travail des décideurs tant publics que privés à Boston et Pittsburgh nous semble reposer sur l'idée que seule une économie forte est capable d'entraîner des retombées sociales pour les exclus. L'effet levier de la croissance sur l'emploi, permettrait, «si les exclus le désirent», de s'arrimer au train de la croissance. Pour qu'une métropole se dote d'une telle croissance, il lui faut mettre en place des conditions qui appuient et supportent un «bon fonctionnement du marché». Dans le cadre de cette logique, il s'agit avant tout d'implanter une gouvernance éclairée, c'est-à-dire capable de mettre à profit un partenariat gagnant entre les entreprises privées, les structures publiques et les institutions d'enseignement supérieur qui mise sur la compétitivité, la productivité et l'innovation. Dans le cas de ces métropoles, il est difficile pour les organismes de développement communautaire de souscrire à une telle approche, même si le financement accordé provient en grande partie de multinationales, via leurs fondations, et de l'État fédéral. D'abord, ces organismes ne sont pas perçus comme des lieux gagnants susceptibles de favoriser une revitalisation de l'économie. Leur image est plutôt celle de structures locales permettant de prévenir une aggravation des problèmes de logement et d'emplois, et, conséquemment, de réguler certaines tensions. Ensuite, cela ne correspond pas à leur mission initiale qui se définit avant tout sur le terrain du social et de la solidarité.
L'analyse du lien entre le développement économique communautaire et les institutions métropolitaines ou municipales à Toronto révèle une faible intégration de la logique du DÉC aux stratégies de développement métropolitain. Ce constat ne signifie pas que les activités de développement économique communautaire n'existent pas à Toronto. Au contraire, notre recherche nous a permis de faire remonter l'émergence de cette stratégie d'intervention au moins au début des années 1980. Par contre, en termes politiques, cette stratégie n'a pas été en mesure de transformer la culture de gouvernance municipale ou métropolitaine, en y intégrant des représentants du milieu communautaire. Le partenariat qu'on retrouve à Toronto est avant tout de type exclusif, c'està-dire public-privé. De plus, les initiatives de DÉC, très fragmentées et portant davantage sur des «communautés d'intérêts » que sur des «communautés locales », ont été incapables de produire des outils politiques de revendication permettant de confronter les dispositifs institutionnels de la métropole torontoise. Cela s'est traduit par une incapacité à porter au-delà de la sphère publique la gestion politique des activités de re-développement et de lutte à l'exclusion.

Néanmoins, la ville de Toronto a implanté différents programmes pour appuyer des initiatives provenant de diverses communautés. Mentionnons les programmes créés par les départements de planification communautaire et de services aux quartiers, par celui de la santé ainsi que par le bureau de développement économique de la Ville. De plus, les organisations de développement économique communautaire reçoivent un financement en provenance tant du palier fédéral que du palier provincial. Ce financement est partagé entre différents ministères dont ceux liés à l'emploi et au développement économique.

Les corporations de développement économique communautaire ont été mises en place à Montréal, au milieu des années 1980, à partir de financements en provenance principalement du gouvernement provincial. Au début des années 1990, la Ville de Montréal a rendu public un plan d'action visant le soutien aux initiatives de développement local issues d'organisations communautaires. Sept corporations de développement économique communautaires qui interviennent à l'échelon de l'arrondissement ont alors été reconnues par la municipalité, tandis qu'elles sont financées par les trois ordres de gouvernement. Depuis la fin des années 1990, ces CDÉC assument les mandats de Centres locaux de 
développement (CLD). Il s'agit de nouveaux organismes de développement mis en place par le gouvernement du Québec dans le cadre d'une politique de soutien au développement local et régional. Au début des années 2000, sur les onze CDÉC de l'île de Montréal et de la nouvelle Ville de Montréal, dix cumulent une mission communautaire à la gestion d'un mandat public.

Sur la scène du développement montréalais, les années 1990 ne sont pas seulement celles de l'institutionnalisation des CDÉC. Elles sont aussi celles de la création de plusieurs organismes de gouvernance et de développement aux échelons de l'île et de la région métropolitaine de Montréal. En 1994, le Conseil régional de développement de l'île de Montréal (CRDÎM) est mis sur pied à la suite d'une politique de développement régional du gouvernement provincial concernant l'ensemble du Québec. Le CRDÎM rassemble des élus provinciaux et municipaux et des représentants du milieu socio-économique, y compris des organismes communautaires. Son principal mandat est d'élaborer un plan stratégique de développement pour l'île de Montréal. Parmi les axes de développement auxquels s'articule le plan stratégique, il y en a un qui se réfère au développement social, à l'économie sociale et à l'action communautaire. À noter, les CDÉC sont intervenues dans l'élaboration du plan stratégique, mais le CRDÎM constitue davantage un forum de discussion qu'une instance de pouvoir.

En 1997, deux autres organismes de développement sont créés à l'initiative, cette fois, des municipalités et du milieu des affaires de la région métropolitaine. Il s'agit de Montréal International dont le mandat est de promouvoir la métropole sur le marché international et Montréal Technovision qui a pour mission de mettre en valeur le potentiel technologique de la métropole. Ces deux organismes ont des liens avec les grandes entreprises et les pouvoirs publics, mais n'ont pas établi de relations avec les CDÉC. Enfin, à l'automne 2000, le gouvernement du Québec a créé la Commission métropolitaine de Montréal dont une des compétences est le développement économique de la région métropolitaine. Toutefois, il est encore trop tôt pour évaluer son arrimage avec les initiatives locales de développement puisque cette commission n'est entrée en opération qu'en 2001.

\section{Conclusion}

La faible prise en considération par les acteurs métropolitains des interventions mises de l'avant par les acteurs du développement économique communautaire est révélatrice des défis à relever pour assurer un emboîtement des stratégies de développement orientées vers les marchés mondiaux dans une perspective de croissance et de celles arrimées aux territoires inframunicipaux et fondées sur des préoccupations sociales.

Notre étude indique que des blocages apparaissent très clairement au niveau de l'espace politique métropolitain. Ce dernier continue de penser le développement comme il était pris en considération sous le fordisme où la division des responsabilités faisait reposer les mesures providentielles sous la juridiction de l'État-nation.

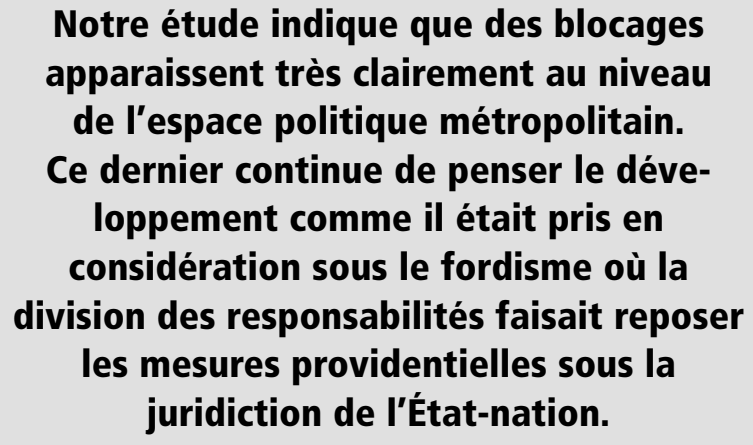

La reconfiguration récente des fonctions de l'État-nation repose sur le questionnement de la centralité occupée par l'État en égard aux mesures povidentialistes. Tant le secteur privé que le secteur communautaire, en passant par les instances décentralisées et déconcentrées de gestion de la sphère publique, sont interpellés par cette recomposition des fonctions de l'État. Le renouvellement des arrangements institutionnels exige des paliers métropolitains qu'ils deviennent plus actifs dans la mise en synergie des capacités d'intervention des différents acteurs présents sur leur territoire.

Notre étude démontre que les acteurs métropolitains demeurent conservateurs et, jusqu'à un certain point, qu'ils préfèrent jouer le jeu de la concurrence territoriale 
pour attirer du développement que d'adopter une culture politique qui miserait sur une vision intégrée du développement de leur territoire. Il découle de cette situation que la scène politico-administrative métropolitaine se laisse bercer par la logique de marché qui repose avant tout sur la capacité d'attirer des investissements, la gestion de l'exclusion socio-économique étant reléguée à la scène communautaire locale. À cet égard, les quatre agglomérations étudiées ne semblent pas échapper au processus de dualisation socio-économique qui affecte les grandes métropoles mondiales.

\section{Notes et références}

1 Cet article est une version remaniée du texte publié dans Géographie, économie et société, volume 4, no. 3, septembre 2002, pp. 305 à 321.

2 Jean-Marc Fontan est professeur au Département de sociologie de l'UQAM; Pierre Hamel est rattaché au Département des sciences humaines appliquées de l'Université de Montréal, Richard Morin au Département d'études urbaines et touristiques de l'UQÀM et Eric Shragge, à la School of Community and Public Affairs de l'Université Concordia.

3 Cette recherche a été rendue possible suite à l'obtention d'un financement du Conseil de recherche en sciences humaines du Canada. La recherche a été réalisée entre 1998 et 2001. 